\title{
Results of head and neck burn reconstructive plastic surgery
}

\author{
Abdurashid Abdusalomovich KAYUMKHODJAEV and Mukhabbat Kuralbaevna KHALMURATOVA ${ }^{\star 凶}$ \\ Republican Specialized Scientific and Practical Medical Center for Surgery named after academician V.Vakhidov", Tashkent, Uzbekistan \\ Corresponding author's Email: muhabbat.phd@gmail.com; (DORCiD: 0000-0002-9258-1660
}

\section{ABSTRACT}

Aim. The present research aimed to analyze the results of all reconstruction types (microsurgical and axial flaps) in head and neck post-burn cicatricial defects. Methods. The study included 114 patients who underwent flaps autotransplantation with microvascular anastomoses, options for plasty with axial flaps on a preserved (natural) vascular pedicle, plasty with displaced flaps, and free skin plasty for the period from 1995 to 2021 . According to the formed groups for comparative analysis, all patients were divided into a comparison group included of 51 patients (1995-2008 year) and the main group with 63 patients (20092021 year). Women predominated with $55.6 \%$ and $58.8 \%$ in the main and comparison groups, respectively. Medium defects $\left(50-120 \mathrm{~cm}^{2}\right)$ of the upper face and neck area prevailed in equal numbers (11.8\%). Most of the patients had a median-lateral unilateral post-burn cicatricial deformity. In terms of functional impairments, grade I up to an angle of $90^{\circ}$ and degree II up to an angle of $45^{\circ}$ was prevailed. Results. The consolidated long-term results of plastic surgery in the functional value were good in the main group in $69.9 \%$ of cases, while in the comparison group it was in $47.1 \%$. The aesthetic indicators of the consolidated long-term results of plastic surgery were good in $57.5 \%$ of cases of the main group and significantly was lower in the comparison group (33.3\% of cases). Unsatisfactory results were observed in $1.4 \%$ of cases in the main group and $17.6 \%$ in the comparison group. Conclusion. Improvement in head and neck burn reconstructive plastic surgery with various variants of axial flaps made it possible to increase the proportion of uncomplicated postoperative course, shorten the hospital stay and the total duration of all stages of plasty, and, in general, ensured a decrease in the frequency of unsatisfactory functional and aesthetic

Research Article

PII: S225199392100014-11

Rec. 24 September 2021

Rev. 13 November 2021

Acc. 19 November 2021

\section{Keywords}

Head and neck burns,

Post-burn cicatricial

defects,

Reconstructive plastic

surgery results.

\section{INTRODUCTION}

Patients with extensive head and neck post-burn lesions belong to the most difficult category, when the postponed thermal injury leads to combined functional and, most importantly, cosmetic complications $[1,2]$. It is the combination of such factors that causes severe psychosomatic disorders, which further aggravates the status of patients.

Reconstructive microsurgery is characterized by an emphasis on the problems of the modern techniques effectiveness in using local tissues for the restoration of extensive defects of the head, neck and upper extremities with lesions of various origins and determining the most promising directions for the development of these technologies $\left[\begin{array}{ll}1 & 2\end{array}\right]$. A number of authors associate the occurrence of such complications as microanastomosis thrombosis, marginal flap necrosis, sub-flap suppuration, suture divergence and rejection with changes in the properties of autologous tissue after implantation and come to the conclusion that the use of musculocutaneous displaced flaps on the vascular pedicle is limited by the length of the vascular pedicle and their large thickness $[2,3,4]$. Free flaps on microvascular anastomoses are devoid of these drawbacks, which contributes to the wider introduction of microsurgical techniques into clinical practice.

Today in science there are various information about the essence of reconstructive treatment and the timing of its beginning. The standard in our country and abroad is reconstructive and restorative operations, which are carried out only after the complete formation of scar tissue, i.e. 8-12 months or more after injury [2, 3 , 4]. Injuries to the soft tissues of the head and neck, for the most part, are extensive, therefore, in order to avoid disruption of forms and functions, and the possibility of recovery with the help of local flaps, some type of regional flap is used $[2,4]$. Depending on the defect, the type of regional flap is selected, its size, location, and 
also the internal properties of the regional flap are taken into account. Single flaps of the insular or perforating type are usually chosen due to the improved blood supply $[3,5,6]$.

Studies have shown that the combined use of a large pectoral flap and a vascularized free bone flap from the fibula provides the viability of closing oromandibular defects $[1,7]$. Also, a number of authors note that the use of contralateral vessels in combination of flaps allows for easy preparation, and also appears to be a low complication rate $(10 \%)$ requiring repeated operations $[4,8,9]$. The main reasons for unsatisfactory and complications are ambiguous, open specific questions remain the choice of the optimal method for the one-step closure of such defects, where a certain place will be occupied by developments in the formation of preprepared (prefabricated) complex (soft tissue-bone or soft tissue-cartilaginous) flaps on the vascular pedicle [10, $11,12]$.

The article presents an analysis of all types of applied reconstructions of the head and neck post-burn defects (plastics by transplanting flaps with the imposition of microsurgical anastomoses and plasty with axial flaps on a natural vascular pedicle).

\section{MATERIALS AND METHODS}

The review board and ethics committee of RSCS named after acad. V.Vakhidov approved the study protocol and informed consents were taken from all the participants.

A total of 114 patients were included, who underwent the indicated variants of plastic surgery in the period from 1995 to 2021. All patients were divided into a comparison group with 51 patients (1995-2008 year) and the main group with 63 patients (2009-2021 year).

In the main group, 10 patients underwent plastic surgery with double flaps, which increases the total number of reconstructive interventions (based on the number of flaps) to 73 , in connection with which, in some analytical tables and graphs, the calculated indicators will be determined from the number of flaps used. In particular, plastic surgery with autologous transplantation on microanastomoses was performed in 22 patients, of whom 3 underwent plastic surgery with double turaco-dorsal flap (TDF) (a total of 6 flaps in 3 patients) for the reconstruction of the cicatricial defect of the head, and also in 2 patients, plastic surgery with double scapular flap (SF) was performed to reconstruct the neck defect, which increased the total number of transplanted flaps in the main group is up to 27.

During plasty with axial flaps with its own blood flow (on a natural pedicle), out of 41 out of 41 , 3 patients underwent plasty with double temporal-temporal flap (TTF) to correct the post-burn scar process in the region of the eyebrows and eyelids, and 2 patients underwent plasty of the neck defect with double epaulet flap (EF), which in general increased the number of flaps to 51 . Also in the main group, plastic techniques were used for the first time with complex soft tissue-cartilaginous flaps of soft tissue injuries of the head and neck, combined with cartilage defects (nose, trachea, auricle).

In all age groups, women prevailed with $55.6 \%$ in the main group, and $58.8 \%$ in the comparison group, while men accounted for $44.4 \%$ in the main group, and $41.2 \%$ in the comparison group. It should be noted that the majority of patients in the main group at the age of $20-44$ years was $44.4 \%$ and at the age of $45-59$ years was 28.6\%; while in the comparison group for patients with $20-44$ years old it was $45.1 \%$ and for those with $45-59$ years old it was $29.4 \%$.

With the distribution of patients by zones and the nature of the defect in the comparison group, medium defects $\left(50-120 \mathrm{~cm}^{2}\right)$ of the upper face and neck area prevailed in equal numbers (11.8\%), in the main group $11.1 \%$ of lesions were in the upper face area and $6.3 \%$ on the neck. Large defects $\left(>120 \mathrm{~cm}^{2}\right)$ of the neck in the comparison group amounted to $35.3 \%$, and in the main group to $39.7 \%$ (Table 1 ).

The indications for the use of microsurgical autotransplantation of flaps in post-burn cicatricial deformities and defects of the head and neck were as follows: deep medium and large cicatricial deformities and defects in cicatricial lesions of the areas of potential axial flaps; extensive deep post-burn cicatricial defects of two adjacent areas of the head (cranial vault - facial area), neck (anterior neck area with a transition to the back); medium and large cicatricial defects with an infected wound, inflammation of the underlying structures (osteomyelitis, chondritis); extensive infected post-burn wound defect of two or more adjacent areas of the head, with necrosis of the underlying structures (cortical bone layer, cartilage, eyeball, auricle), with exposed cavities (maxillary sinus, orbit), requiring simultaneous plastic surgery.

The indications for the use of axial flaps in the aftermath of deep extensive burns of the head and neck were as follows: medium and large post-burn cicatricial defects of the head and neck, the possibility of closure 
of which by local tissues is limited; medium and large deep post-burn cicatricial defects and deformities of the head and neck in the absence of the possibility of using microsurgical flaps due to the absence of recipient vessels; open cavities (through cicatricial defects) of the skull, upper jaw, neck with a large defect in the tracheal wall, in the absence of recipient vessels for revascularization of the microsurgical flap; in the absence of conditions for performing microsurgical autotransplantation of flaps (specialists, equipment, etc.).

Table 1. Distribution of patients by zones and nature of the defect

\begin{tabular}{|c|c|c|c|c|c|c|}
\hline \multirow[t]{2}{*}{ Zone of the defect } & \multicolumn{2}{|c|}{$\begin{array}{l}\text { Medium defects } \\
\left(50-120 \mathrm{~cm}^{2}\right)\end{array}$} & \multicolumn{2}{|c|}{$\begin{array}{l}\text { Large defects } \\
\qquad\left(>120 \mathrm{~cm}^{2}\right)\end{array}$} & \multicolumn{2}{|c|}{ Total } \\
\hline & $\mathbf{n}$ & $\%$ & $\mathbf{n}$ & $\%$ & $\mathbf{n}$ & $\%$ \\
\hline \multicolumn{7}{|l|}{ Comparison group } \\
\hline Upper face area & 6 & $11.8 \%$ & 1 & $2.0 \%$ & 7 & $13.7 \%$ \\
\hline Middle zone of the face & 0 & $0.0 \%$ & 3 & $5.9 \%$ & 3 & $5.9 \%$ \\
\hline Lower face area & 1 & $2.0 \%$ & 2 & $3.9 \%$ & 3 & $5.9 \%$ \\
\hline The defeat of several areas of the face & 2 & $3.9 \%$ & 9 & $17.6 \%$ & 11 & $21.6 \%$ \\
\hline Calvaria & 0 & $0.0 \%$ & 3 & $5.9 \%$ & 3 & $5.9 \%$ \\
\hline Neck & 6 & $11.8 \%$ & 18 & $35.3 \%$ & 24 & $47.1 \%$ \\
\hline Total & 15 & $29.4 \%$ & 36 & $70.6 \%$ & 51 & $100.0 \%$ \\
\hline \multicolumn{7}{|l|}{ Main group } \\
\hline Upper face area & 7 & $11.1 \%$ & 2 & $3.2 \%$ & 9 & $14.3 \%$ \\
\hline Middle zone of the face & 0 & $0.0 \%$ & 2 & $3.2 \%$ & 2 & $3.2 \%$ \\
\hline Lower face area & 2 & $3.2 \%$ & 0 & $0.0 \%$ & 2 & $3.2 \%$ \\
\hline The defeat of several areas of the face & 2 & $3.2 \%$ & 14 & $22.2 \%$ & 16 & $25.4 \%$ \\
\hline Calvaria & 0 & $0.0 \%$ & 5 & $7.9 \%$ & 5 & $7.9 \%$ \\
\hline Neck & 4 & $6.3 \%$ & 25 & $39.7 \%$ & 29 & $46.0 \%$ \\
\hline Total & 15 & $23.8 \%$ & 48 & $76.2 \%$ & 63 & $100.0 \%$ \\
\hline
\end{tabular}

\section{RESULTS AND DISCUSSION}

Table 2 shows the summary rate of complications after all types of reconstruction of head and neck defects. The most common complications in head and neck defect in the study group are microanastomoses thrombosis (4.1\%), sub-flap suppuration (4.1\%), flaps with complications (9.6\%), as well as patients with complications (11.1 \%). In the comparison group, these indicators are significantly higher ( $\mathrm{p}<0.05)$ : thrombosis of microanastomoses (17.6\%), sub-flap suppuration (11.8\%), and flaps and patients with complications (35.3\%), respectively. In addition, in the comparison group, marginal flap necrosis and suture divergence developed (11.8\%).

Figure 1 shows the data on the distribution of the proportion of complicated postoperative course by groups. Complications of flaps in the comparison group developed in $35.3 \%$ of patients, $9.6 \%$ in the main group, while flaps without complications were in $64.7 \%$ of patients in the comparison group and in $90.4 \%$ in the main group. Complications developed in $35.3 \%$ of patients in the comparison group, without complications in $64.7 \%$ of patients. In the main group, these indicators were $11.1 \%$ (with complications) and $88.9 \%$ (without complications), respectively.

Table 3 summarizes the data on the need for corrective interventions. In $63.0 \%$ of patients in the comparison group, corrective intervention was required after the operation of the head defect, and in the main group was $40.0 \%$. After correction of neck defects in the comparative group, $41.7 \%$ were corrected, and in the main group it was $18.2 \%\left(\chi^{2}=6.533 ; \mathrm{df}=1 ; \mathrm{p}=0.011\right)$. Reconstruction surgery (flap necrosis) in the comparative group was performed in $9.8 \%$ of patients, and in the main group, only $1.4 \%$ of patients required additional intervention. Repeated interventions (stage 1) in the group were compared in $13.7 \%$ of patients, while, as in the main group, corrective interventions were required only in $6.8 \%$. It should be noted that in the comparison group in $64.7 \%$ of cases, a second operation was not required, and in the main group this indicator was much better (90.4\%) (Table 4). Taking into account the fact that VTL was used for lesions of the eyebrow and eyelid zones and did not require closure of other adjacent zones in the structure of axial flaps, the summary data of this type of plasty are presented separately. 
Table 2. Summary frequency of complications after all types of reconstruction of head and neck defects

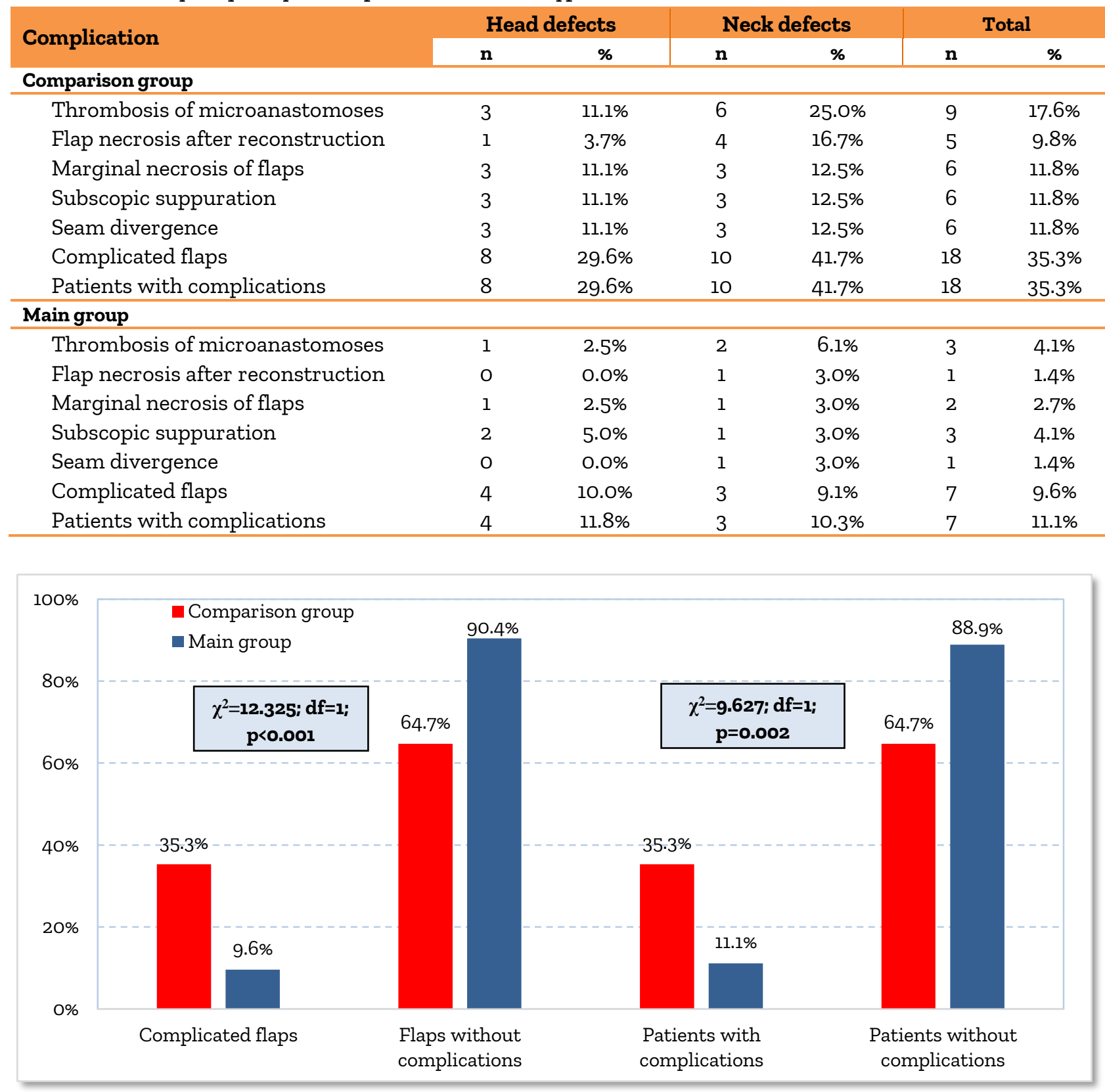

Figure 1. Distribution of the proportion of complicated postoperative course by groups

Table 3. The need for corrective interventions (from the number of flaps)

\begin{tabular}{lcc|cc} 
& \multicolumn{2}{c}{ Comparison group } & \multicolumn{2}{c}{ Main group } \\
Defect localization & $\mathbf{n}$ & $\mathbf{\%}$ & $\mathbf{n}$ & $40.0 \%$ \\
After correction of head defects & $17 / 27$ & $63.0 \%$ & $16 / 40$ & $18.2 \%$ \\
After correction of neck defects & $10 / 24$ & $41.7 \%$ & $6 / 33$ & $30.1 \%$ \\
All defects & $27 / 51$ & $52.9 \%$ & $22 / 73$ & \\
$\chi^{2}$ & & $6.533 ; \mathrm{df}=1 ; \mathrm{p}=0.011$ & \\
\hline
\end{tabular}

Table 4. Distribution of patients by the number of reconstructions and stages of corrective operations (from the number of flaps)

\begin{tabular}{lcc|cc} 
& \multicolumn{2}{c}{ Comparison group } & \multicolumn{2}{c}{ Main group } \\
Resurgery & $\mathbf{n}$ & $\mathbf{\%}$ & $\mathbf{n}$ & \multicolumn{1}{c}{$1.4 \%$} \\
Reconstruction (flap necrosis) & $5 / 51$ & $9.8 \%$ & $1 / 73$ & $6.8 \%$ \\
Correction in 1 stage & $7 / 51$ & $13.7 \%$ & $5 / 73$ & $1.4 \%$ \\
Correction in 2 stages & $6 / 51$ & $11.8 \%$ & $1 / 73$ & $90.4 \%$ \\
No repeated interventions & $33 / 51$ & $64.7 \%$ & $66 / 73$ & \\
$\chi^{2}$ & & $15.450 ; \mathrm{df}=3 ; \mathrm{p}=0.002$ & \\
\hline
\end{tabular}

Citation: Kayumkhodjaev AA, and Khalmuratova MK. Results of head and neck burn reconstructive plastic surgery. J Life Sci Biomed, 2021; 11(6): 113-119. DOI: https://dx.doi.org/10.51145/jlsb.2021.14 
The operation with microsurgical flaps, carried out in stage 1, was used in the comparative group in $47.4 \%$ of patients, and the indicators of the main group were better (81.8\%). Intervention in 2 stages was required in the comparative group in $36.8 \%$ of patients, and intervention in 3 stages in the comparative group was performed in $15.6 \%$ of patients. In the main group, the indicator was 9.1\%, with operations in stages 2 and 3 . Axial and deltopectoral and epolent flaps in 3 stages were performed in the comparative group in $44.0 \%$ of patients, and in the main group - 57.6\%. 3-stage axial temporal flaps were used both in the comparison group (100\%) and in the main group (100\%). The frequency of using operations in several stages was compared in the study groups (Figure 2). So, most of all operations were performed in 3 stages, and in the comparative group in $41.2 \%$, in the main group in $46.0 \%$. Five-stage operations in the comparative group were performed in $5.9 \%$, and for the patients of the main group, they were not performed (o\%).

The length of hospital stay for all stages of reconstruction was, $25.2 \pm 8.0$ days in comparison, and $20.7 \pm 6.6$ days in the main group - $(\mathrm{t}=3.25 ; \mathrm{p}<0.001)$. At the same time, the total duration of all stages of plastic surgery was also shorter in the main group of patients, amounting to $43.9 \pm 26.6$ days versus $70.2 \pm 42.3$ days in the comparison group ( $t=3.88 ; \mathrm{p}<0.001)$.

Long-term results were followed in all patients. A good functional result for head defects was shown both in the comparative group (51.9\%) and in the main group (72.5\%). There is no unsatisfactory functional result in the main group (o\%). With neck defects, good indicators were observed in the comparative group in $41.7 \%$ of patients, and in the main group in 66.7\%. A good functional result with head defects was presented in the main group in $62.5 \%$, and in the comparative in $33.3 \%$. The best aesthetic result for head defects was registered among the patients of the main group there were no unsatisfactory results (o\%).

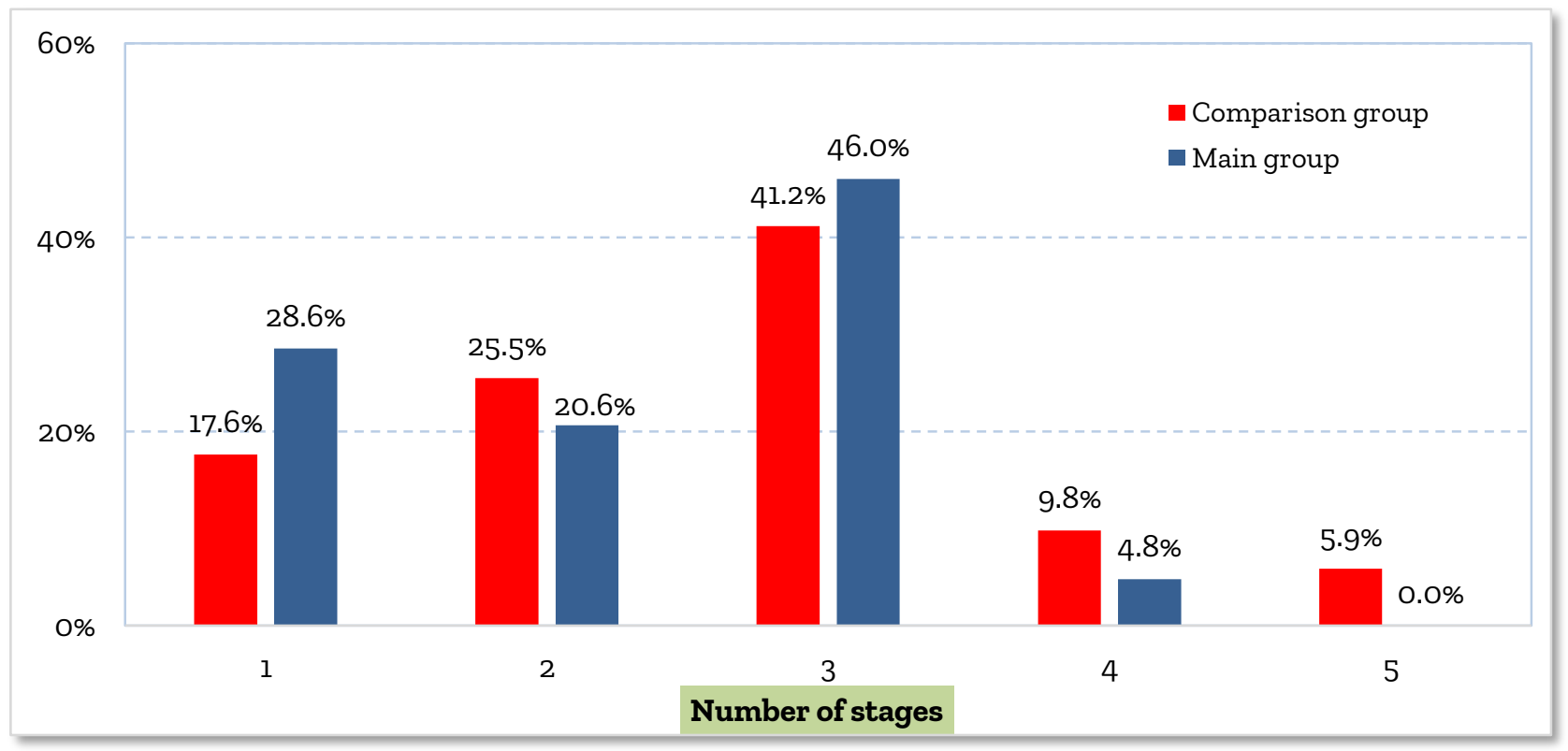

Figure 2. Summary distribution of patients by the number of all stages of plastic surgery

The summary long-term results of plastic surgery were good, in a functional value, in the main group in 69.9\% of cases (Figure 3), and in the comparison group in 47.1\%. Satisfactory in the comparison group was $35.3 \%$, and in the main group was $28.8 \%$. Unsatisfactory results were observed in the main group in $1.4 \%$ of cases, and in the comparison group they were $17.6 \%\left(\mathrm{Df}=2, \mathrm{p}=0.002, \chi^{2}=12.852\right)$. Aesthetic indicators of the consolidated longterm results of plastic surgery were good, 57.5\% of cases in the main group, and 33.3\%, in the comparison group (significantly lower) Satisfactory in the comparison group was 47.1\%, and in the main group was $41.1 \%$. Unsatisfactory results were $1.4 \%$ of cases in the main group, $17.6 \%$ in the comparison group $(\mathrm{df}=2, \mathrm{p}<0.001$, $\left.\chi^{2}=13.843\right)$.

Also, during the study, it was determined that the tactics of surgical treatment, as well as indications for one or another type of plastic surgery for post-burn cicatricial defects and deformities of the head and neck depend on the following factors: the area and depth of the cicatricial defect, the state of the surrounding tissue defect; the nature of the scars, namely their maturity, the type of scars (normotrophic, hypo- or hypertrophic, keloid); condition of tissues of potential axial flaps in areas adjacent to the defect; the presence of infection, necrosis (osteomyelitis) of tissues in the area of the post-burn cicatricial defect; the degree of scar deformity or defect in the 
underlying anatomical structure (auricle, nose, lips, eyelids, eyebrows, trachea); tissue deficiency, which causes contracture or contracting deformity of the neck; the degree of functional disorders, depending on which further tactics of surgical treatment will be chosen; the condition of the recipient vessels used for revascularization of the microsurgical flap; the severity of the aesthetic defect; the preparedness of the institution, as well as the availability of specialists to perform one or another method of plastics.
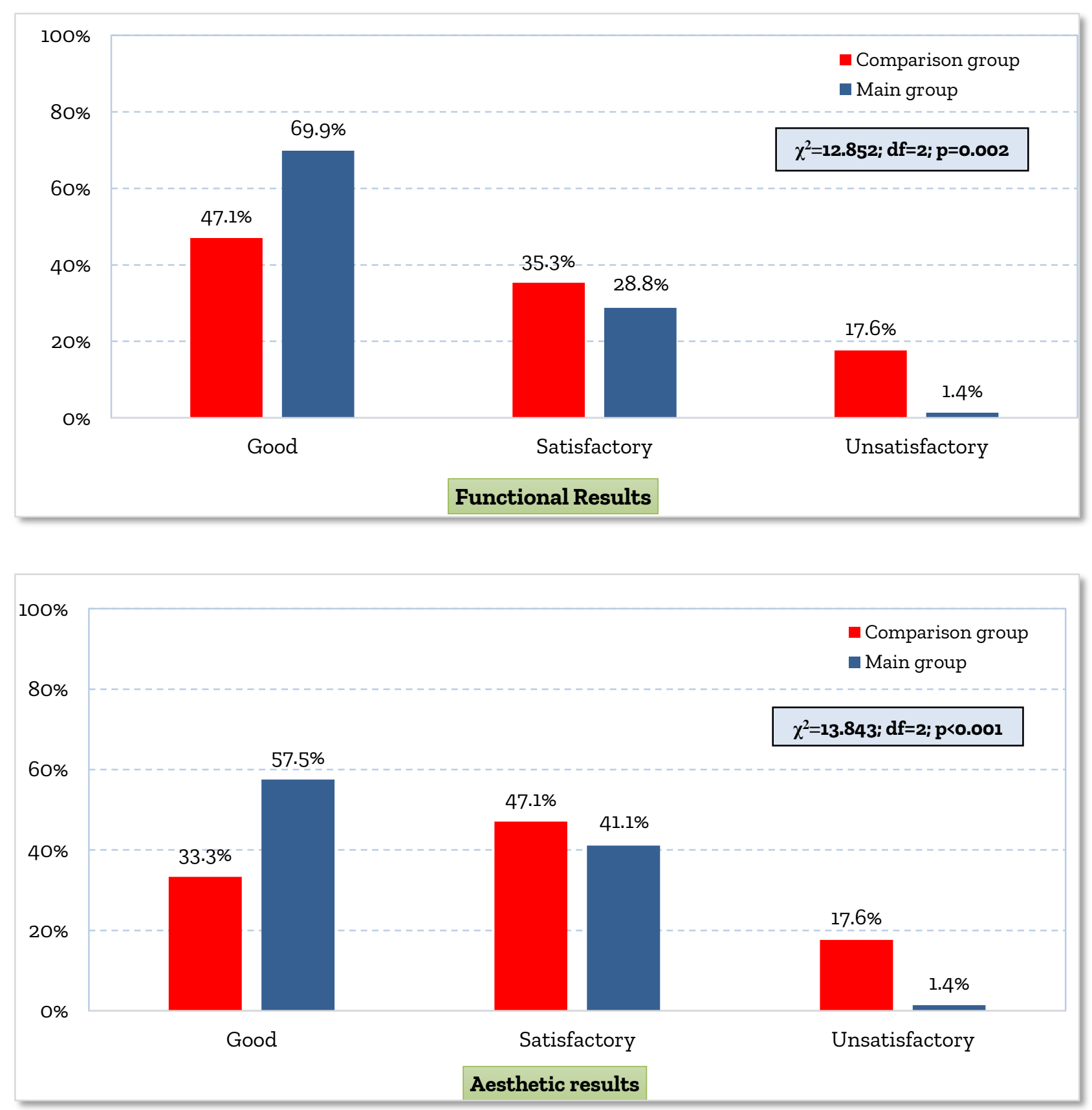

Figure 3. Summary of long-term results of plastic surgery

\section{CONCLUSION}

Formation of axial flaps for the reconstruction of cicatricial defects of the head and neck is technically simpler, does not require additional equipment and materials; therefore their use is preferable. The presence of a sufficient number of donor zones of axial flaps near the defects of the head and neck, provided they are preserved, allows them to be widely used for plastic surgery in conventional surgical departments, including regional multidisciplinary medical centers. Improvement of the tactical and technical aspects of plasty of post-burn defects of the head and neck with various variants of axial flaps made it possible to increase the proportion of 
uncomplicated postoperative course, shorten the hospital stay and the total duration of all stages of plasty, and, in general, ensured a decrease in the frequency of unsatisfactory functional and aesthetic results.

\section{DECLARATIONS}

Acknowledgements

This work was supported by Republican Specialized Centre of Surgery, Tashkent City, Uzbekistan.

\section{Authors' contributions}

All authors contributed equally to this work.

\section{Competing interests}

The authors declare that they have no competing interests.

\section{REFERENCES}

1. Qaisi M, Dee R, Eid I, Murphy J, Velasco Martinez IA, Fung H. Reconstruction for Complex Oromandibular Facial Defects: The Fibula Free Flap and Pectoralis Major Flap Combination. Case Rep Surg. 2019 Mar 26; 2019:8451213. DOI: https://doi.org/10.1155/2019/8451213 ; PMID: 31049242; PMCID: PMC6458920.

2. Schellenberg $\mathrm{M}$, Owattanapanich N, Grigorian A, Lam L, Nahmias J, Inaba K. Surviving Nonsurvivable Injuries: Patients Who Elude the 'Lethal' Abbreviated Injury Scale (AIS) Score of Six. J Surg Res. 2021 Aug 29; 268:616-622. doi: https://doi.org/10.1016/j.jss.2021.06.087; Epub ahead of print. PMID: 34469860.

3. Coleman SR, Lam S, Cohen SR, Bohluli B, Nahai F. Fat Grafting: Challenges and Debates. Atlas Oral Maxillofac Surg Clin North Am. 2018 Mar; 26(1):81-84. doi: https://doi.org/10.1016/j.cxom.2017.10.006 ; PMID: 29362076.

4. Mochizuki Y, Harada H, Shimamoto H, Tomioka H, Hirai H. Multiple Free Flap Reconstructions of Head and Neck Defects Due to Oral Cancer. Plast Reconstr Surg Glob Open. 2017 Jun 13:5(6):e1337. doi: https://doi.org/10.1097/GOX.0000000000001337; PMID: 28740764; PMCID: PMC5505825.

5. Frederick JW, Sweeny L, Carroll WR, Peters GE, Rosenthal EL. Outcomes in head and neck reconstruction by surgical site and donor site. Laryngoscope. 2013 Jul; 123(7):1612-7. doi: https://doi.org/10.1002/lary.23775 ; Epub 2013 May 17. PMID: 23686870; PMCID: PMC3695001.

6. Masia J, Olivares L, Koshima I, Teo TC, Suominen S, Van Landuyt K, et al. Barcelona consensus on supermicrosurgery. J Reconstr Microsurg. 2014 Jan;30(1):53-8. doi: https://doi.org/10.1055/s-0033-1354742 ; Epub 2013 Sep 13. PMID: 24037459.

7. Eskander A, Kang SY, Teknos TN, Old MO. Advances in midface reconstruction: beyond the reconstructive ladder. Curr Opin Otolaryngol Head Neck Surg. 2017 Oct; 25(5):422-430. doi: https://doi.org/10.1097/M00.0000000000000396 ; PMID: 28692450.

8. Kania K, Chang DK, Abu-Ghname A, Reece EM, Chu CK, Maricevich M, Buchanan EP, Winocour S. Microsurgery Training in Plastic Surgery. Plast Reconstr Surg Glob Open. 2020 Jul 17;8(7):e2898. doi: https://doi.org/10.1097/GOX.0000000000002898; PMID: 32802641; PMCID: PMC7413759.

9. Wei FC, Tay SK. Principles and techniques of microvascular surgery. In: Neligan PC, editor. Plastic Surgery. Vol. 1 , Principles. 3rd ed. Elsevier; 2013. 587-621. Google Scholar

10. Almobarak BA, Mohamed M, Hassan M, Kotb M. Regional Flaps in Coverage of Facial Defects: Our Experience. Egypt J Plast Reconstr Surg. 2018; 42(1): 45-53. Corpus ID: 52209121

11. Ray E. Head and Neck Reconstructive Surgery. Cancer Treat Res. 2018; 174:123-143. doi: https://doi.org/10.1007/978-3319-65421-8 8; PMID: 29435840.

12. Murphy BD, Kerrebijn I, Farhadi J, Masia J, Hofer SOP. Indications and Controversies for Abdominally-Based Complete Autologous Tissue Breast Reconstruction. Clin Plast Surg. 2018 Jan; 45(1):83-91. doi: https://doi.org/10.1016/j.cps.2017.08.007 ; Epub 2017 Sep 19. PMID: 29080663. 